OPEN ACCESS

Edited by:

Li Ang,

First Affiliated Hospital of Zhengzhou

University, China

Reviewed by:

Dhuha Alajmi,

Ministry of Health, Kuwait

Payam Behzadi,

Islamic Azad University, Iran

Lan Yan,

Second Military Medical University, China

${ }^{*}$ Correspondence:

Chun-yan Wang

615916339@qq.com

tThese authors share first authorship

Specialty section:

This article was submitted to Infectious Diseases - Surveillance,

Prevention and Treatment,

a section of the journal

Frontiers in Medicine

Received: 03 February 2021

Accepted: 20 September 2021

Published: 22 October 2021

Citation:

Cao X-g, Yu C-w, Zhou S-s, Huang Y and Wang C-y (2021) Case Report: A

Candida Meningitis in an Immunocompetent Patient Detected

Through the Next-Generation

Sequencing. Front. Med. 8:656066.

doi: 10.3389/fmed.2021.656066

\section{Case Report: A Candida Meningitis in an Immunocompetent Patient Detected Through the Next-Generation Sequencing}

\author{
Xiao-guang $\mathrm{CaO}^{1 \dagger}{ }^{1+}$ Chuang-wei $\mathrm{Yu}^{2 \dagger}$, Shu-sheng Zhou ${ }^{1}, \mathrm{Yu}$ Huang ${ }^{1}$ and Chun-yan Wang ${ }^{1 *}$ \\ ${ }^{1}$ Department of Emergency Intensive Care Unit (EICU), The First Affiliated Hospital of University of Science and Technology of \\ China (Anhui Provincial Hospital), Hefei, China, ${ }^{2}$ Department of Emergency Intensive Care Unit (EICU), TaiHe County \\ People's Hospital, Fuyang, China
}

Background: Fungal infections of the central nervous system (CNS) are not commonly seen clinically. Clinical diagnosis of fungal infections often depend on the pathogen culture and the clinical features. This method is time-consuming and insensitive, which can lead to misdiagnosis. The authors introduce an adult patient with fungal infections diagnosed by next-generation sequencing (NGS).

Case: The patient was a 60-year-old male Chinese who had both hypermyotonia of the lower extremities and fever. The auxiliary examinations such as MRI, CT, and cerebrospinal fluid (CSF) analysis showed obvious abnormalities. Because of the difficulties in diagnosis, it was hard to determine the treatment plan. The NGS detected specific sequences of Candida albicans in 3 days. The patient was then treated with liposomal amphotericin B and fluconazole. About 3 weeks later, the symptoms of the patient improved significantly and he was discharged from the hospital.

Conclusion: Compared with the routine cultural method, NGS has made a huge advancement in infection diagnosis and targeting antimicrobial therapy for CNS infection.

Keywords: next-generation sequencing, central nervous system infections, Candida albicans, fungal infections, antimicrobial therapy

\section{INTRODUCTION}

Fungal infections include Candida infection, cryptococcal infection, Pneumocystis carinii infection. (1). It is well-known that fungal infections are serious opportunistic infectious diseases, although the proportion of patients with fungal infections is not high. Moreover, based on the current epidemiological survey (2), the prevalence of fungal infections is increasing every year. There are at least 15 distinct Candida species that cause human disease, but $>90 \%$ of the invasive disease are caused by the five most common pathogens such as C. albicans, C. glabrata, C. tropicalis, C. parapsilosis, and C. krusei (3).

Recently, invasive fungal infections (IFIs) have gained importance in public health due to the increasing number of patients with immunocompromised who are at risk for the infections caused by these opportunistic pathogens (4), especially the fungal infections of the central nervous system (CNS) (5). 
Because most of these cases of fungal CNS infection are chronic and develop in patients with an altered immune response, it is difficult for clinicians to diagnose early from the clinical manifestation. The development of fungal CNS infections also mainly depends on the interplay between the health status and fungal virulence factors of the patients.

As we know, in clinical cases, the most common fungal pathogens include Cryptococcus spp., Aspergillus spp., Histoplasma capsulatum, and Candida albicans. Candida meningitis is the most common fungal pathogen in CNS in clinical cases (6).

Although these diseases are relatively rare, these lethal infections in the CNS give rise to significant morbidity and mortality. Meanwhile, the diagnosis of the fungal infection is often difficult and the treatment is often delayed. There have been catastrophic results because of the misdiagnosis and difficulty in the early diagnosis $(5,6)$. Patients with cerebrovascular disease are more likely to suffer from the fungal infection of the CNS. The incidence was related to invasive operations, broad-spectrum antibiotics abuse, and the immune system and immunodepression of the patients.

In this case, the pathogens were not found by the cerebrospinal fluid (CSF) and blood culture test. At the same time, we could not get any help from MRI and CT. The treatment of the patients is in a dilemma. Then, the next-generation sequencing (NGS) method was used to detect the pathogen and the result showed that the pathogen was Candida.

\section{CASE}

A 60-year-old male had a history of fever for half-month (January 1, 2019), diarrhea, 1-week history of somnolence, and extremity of hypertonia. Apart from the past medical history of chronic bronchitis and surgery of hernia, we did not get any meaningful history from his family.

When the symptoms began, he had a fever and the highest body temperature of the patient was $39^{\circ} \mathrm{C}$. On the next day, he began to have incontinence and diarrhea. The patient was then sent to the Huainan Xinhua Hospital. The CT of the chest showed that he had pulmonary inflammation; the patient was given the treatment of anti-infection, but the symptoms were not alleviated. About 4 days later, the patient was transferred to the Huainan First People's Hospital and he required the serial lumbar punctures as his intracranial pressure raised persistently. The CSF test showed the abnormal results that CSF protein level and pressure were higher than usual (family members could not provide the accurate results and anti-infective agents). Meanwhile, the body temperature of the patient still fluctuated between 37.5 and $38.1^{\circ} \mathrm{C}$. By using the CSF culture and virus antibodies, no pathogen was detected, so the patient continued to receive empiric treatment for viral meningitis and supportive care. Then, the patient developed the symptoms of cough and phlegm, with paroxysmal fever as high as $38.1^{\circ} \mathrm{C}$. Because the symptoms of the patients did not improve, he was transferred to the Anhui Provincial Hospital. On arrival, the patient was afebrile and had a blood pressure of $127 / 80 \mathrm{~mm} \mathrm{Hg}$ and a heart rate of 72
TABLE 1 | Cerebrospinal fluid test.

\begin{tabular}{lll}
\hline $\begin{array}{l}\text { Open Pressure } \\
\text { Colors }\end{array}$ & $80 \mathrm{mmHg}$ \\
Cell Count & Karyocyte Cell & Transparent \\
& Red Blood Cell & $10 \times 10^{\wedge} 6 / \mathrm{L}$ \\
& Mononuclear Cell & 0 \\
& Multinucleated Cell & $100 \%$ \\
Glucose & & $0 \%$ \\
Protein & & $2.48 \mathrm{mmol} / \mathrm{L}$ \\
Chloride & & $0.66 \mathrm{~g} / \mathrm{L}$ \\
Ig-G & & $115.2 \mathrm{mmol} / \mathrm{L}$ \\
Ig-A & $54.6 \mathrm{mg} / \mathrm{L}$ \\
Ig-M & $9.50 \mathrm{mg} / \mathrm{L}$ \\
\end{tabular}

beats/min. The physical examination showed that he had neck stiffness and hypermyotonia. He had no focal motor or sensor deficits and his nerve examination was normal.

As a result of these data and information, the doctors considered that the patient had viral encephalitis and he was treated with gammopathy, methylprednisolone, and anti-virus therapy. Viral antibodies and $\beta-1,3$ glucanase were used to speculate on the type of pathogen again. Combined with the previous examination results of the patients in the Huainan and the current examination results, we did not get the valuable results to identify the pathogens. Therefore, the patient continued to receive empiric treatment for the viral encephalitis and supportive care, but the symptoms were not alleviated. Due to the tetanus antibody that is probable positive, the patient was transferred to the infectious department (January 13, 2019). According to the clinical symptoms, the doctors did not consider the possibility of tetanus. Then, the patient again returned to the neurology department. When the patient arrived, he showed unconsciousness and his oxygen saturation of blood decreased. For further treatment, the patient was sent to the intensive care unit (ICU) (January 15, 2019). Subsequently, the lumbar puncture was performed again, showing an open pressure of $80 \mathrm{~mm} \mathrm{Hg}$. The CSF test showed glucose of 2.48 $\mathrm{mmol} / \mathrm{l}$, protein of $0.66 \mathrm{~g} / \mathrm{l}$, karyocyte count of $10^{10} \sim 6 / \mathrm{L}$, and chloride of $115.2 \mathrm{mmol} / \mathrm{l}$ (Table 1). Repetitive serology and CSF studies for the possible organisms continued to be negative. Gram staining and India ink staining did not show any organisms. CSF culture was repeated. While MRI of the brain revealed the abnormal enhancement of meningitis and hemangioma, we did not get any valuable information from MRI.

During this period, the C-reactive protein (CRP) of the patient, mental state, and body temperature gradually decreased after the antibacterial treatment. His sample was sent for pathogen detection by the NGS at the Beijing Genomics Institute (BGI), Wuhan, China. CSF sample from the patient was analyzed by using the BGI PMSeq high-throughput sequencing process for the detection of pathogenic microorganisms. DNA from the CSF sample was extracted by using the TIANamp Micro DNA Kit (Beijing, China). Then, $100 \mathrm{ng}$ of extracted 


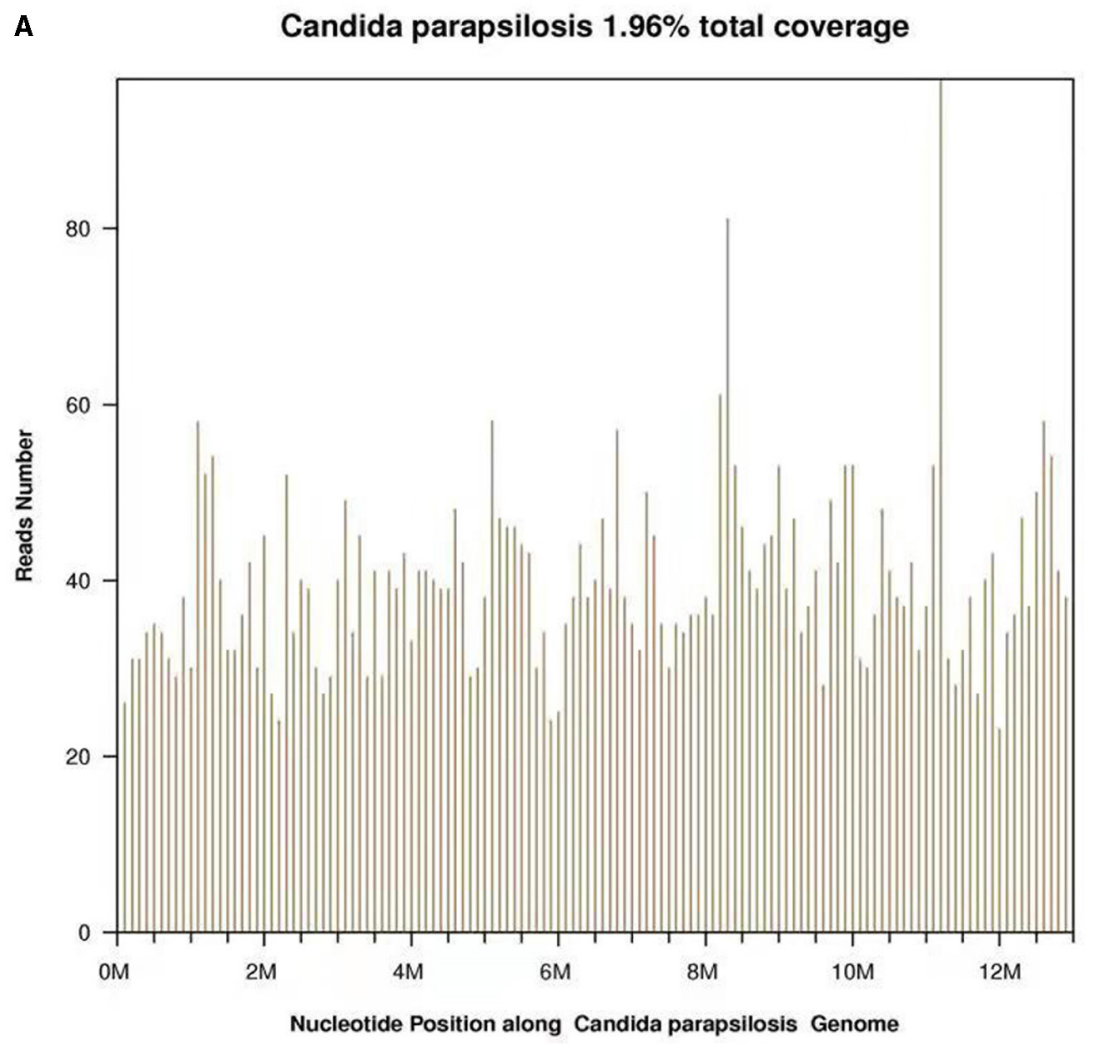

B Candida orthopsilosis $0.2637 \%$ total coverage

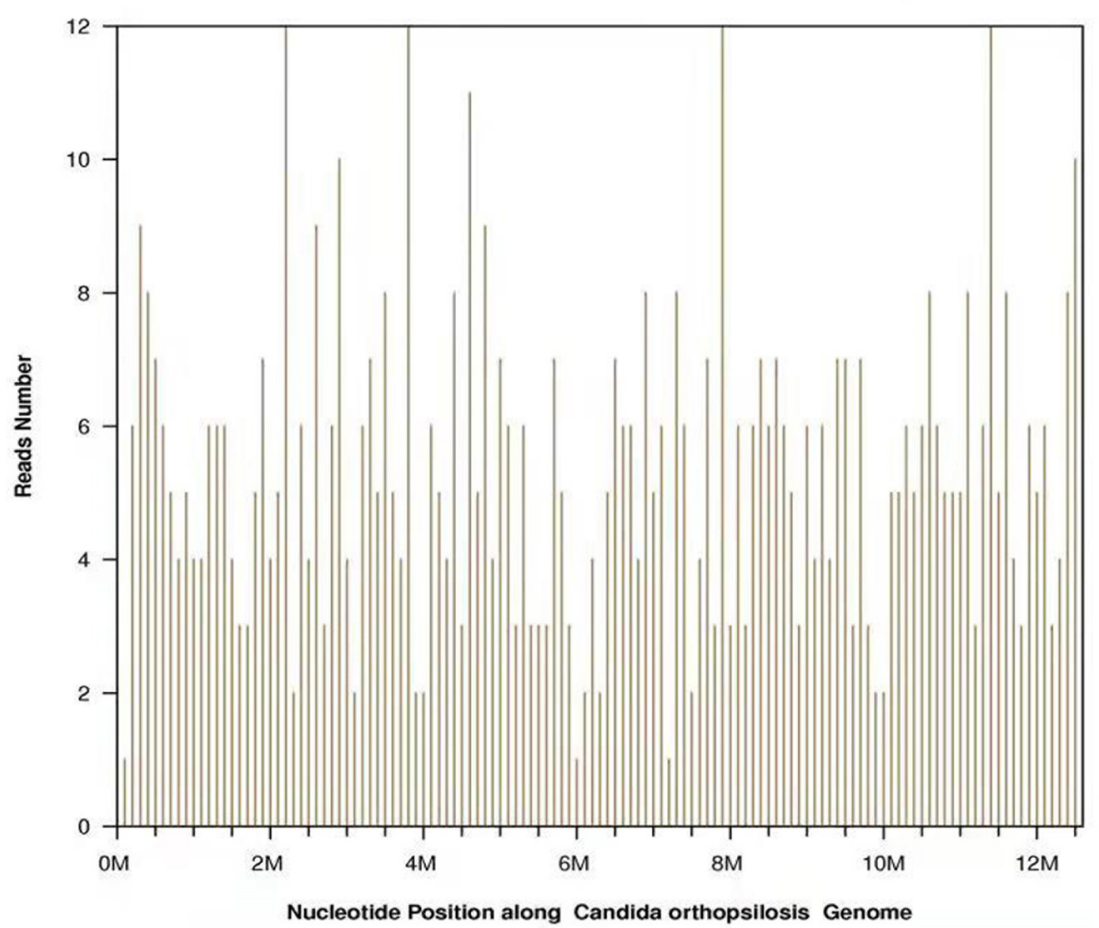

FIGURE 1 | (A) A total of 5,212 reads were mapped to the Candida parapsilosis in the reference database. The coverage of the referenced Candida parapsilosis genome was $1.96 \%$. (B) A total of 675 reads were mapped to the Candida orthopsilosis in the reference database. The coverage of the referenced Candida orthopsilosis genome was $0.2637 \%$. 


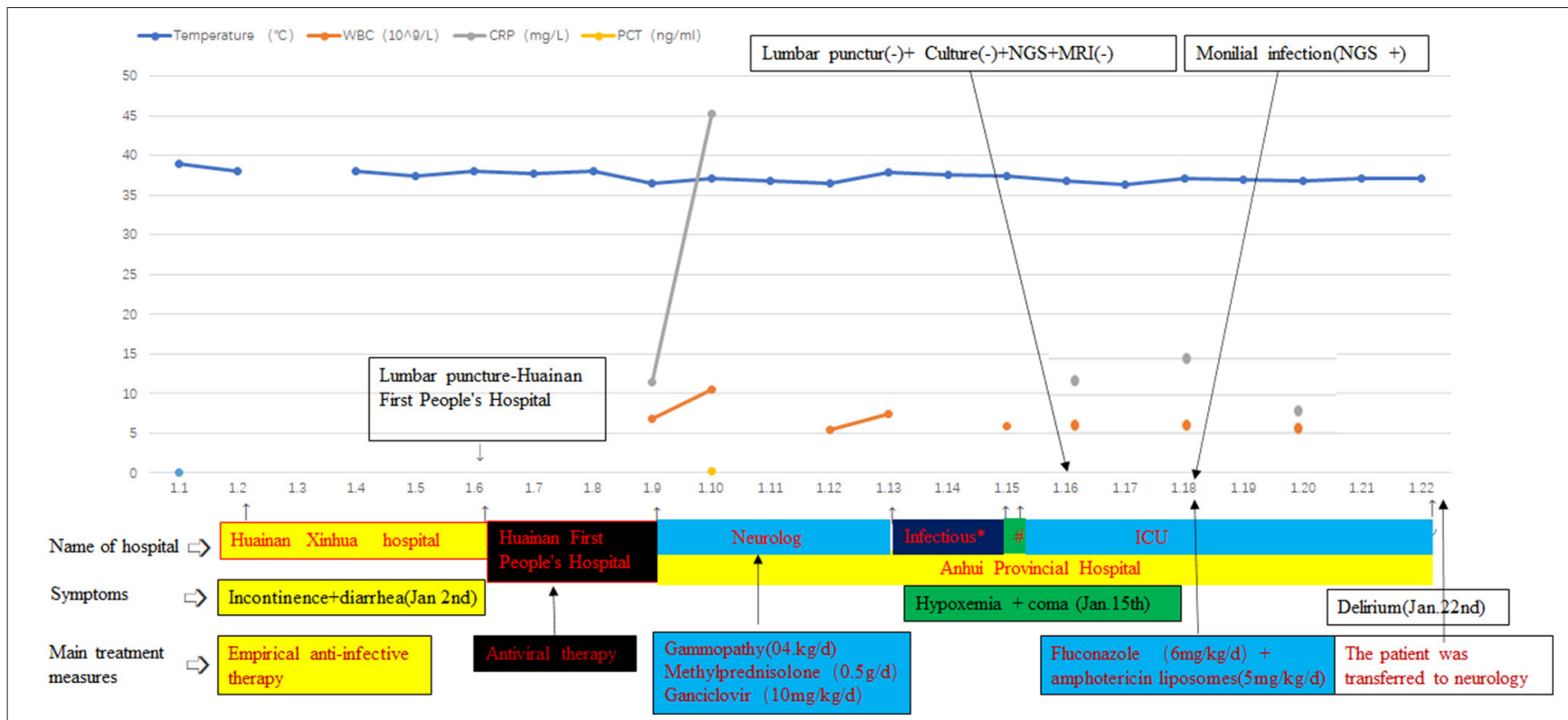

FIGURE 2 | Annotation: "*” indicated the department of infection; "\#” indicated that the patient was treated in the neurology department in the afternoon and he was transferred to the intensive care unit (ICU) in the evening; "-" means negative; and "+" means positive. The beginning and end of each color bar indicated the treatment measures during this period.

DNA were subjected to the library building steps including fragmentation, end repair, and sequencing adapter ligation. Then, the DNA library was sequenced on the BGISEQ-500 sequencing platform at the BGI, Wuhan, China. A total of $3,310,880$ single-end reads were generated. Then, the reads were processed by using the BGI metagenome analysis platform. After filtering of the human genome sequences, the rest sequences were mapped to a metagenome database including 2,328 bacteria, 199 fungi, 4,189 viruses, and 135 parasites from the National Center for Biotechnology Information (NCBI). Among these reads, 30,451 were mapped to Candida parapsilosis (Figure 1A) and 2,857 were mapped to Candida orthopsilosis (Figure 1B), all these are fungal species. The other reads were mapped to the common environmental microbes or laboratory contaminants.

Due to this result, there was a debate among the doctors. Doctors were divided into two groups. One group thought that the foundation of the fungal infection was not sufficient at the current stage and the epidemiological investigation in China showed that the incidence of Candida infection in the CNS was low, so they thought that the possibility of contamination of the specimen cannot be ruled out. Because antithyroglobulin antibodies in the patient were positive, combined with thyroid insufficiency and MRI, they considered the possibility of Hashimoto's encephalitis, and the patient should be treated with the hormones. For these reasons, they believed that antifungal therapy was not essential. In the opinion of the other group, although there was no other evidence of the pathogens at present and some clinical symptoms of the patient improved, his muscle tension was still high. Meanwhile, there was no better detection method in our hospital. According to the Infectious Diseases
Society of America (IDSA) guidelines (recommendations 28, 32, 33 , and 94) (3), the change of the clinical symptoms is the most important basis for the clinicians without basis. Furthermore, the empirical treatment of adults with encephalitis is with drugs within $6 \mathrm{~h}$ of suspicion (7), and delay in the diagnosis and inappropriate therapy would result in permanent damage, even also death. It was an unacceptable outcome. Owing to the poor therapeutic effect and symptoms, diagnostic treatment can be initiated and the neurologists also agreed with the treatment. Then, the patient finally received the antifungal treatment with fluconazole combined with the amphotericin liposomes (the dose of fluconazole on the first day was $12,6 \mathrm{mg} / \mathrm{kg} / \mathrm{day}$; amphotericin liposomes: $5 \mathrm{mg} / \mathrm{kg} /$ day) (3) and the neurologists and the family of the patient agreed with this treatment. The clinicians monitored the side effects of this treatment regimen such as hypokalemia and renal impairment. Fortunately, after 4 days of treatment, there was a significant improvement in the muscle tone without the side effects. Because of the enclosed environment of the ICU, the patient developed some mental symptoms (restlessness and delirium appeared intermittently). Because the family members are not allowed to accompany in the ICU, the patient was transferred to the neurology unit for further treatment. Doctors hope to treat delirium through the company and psychological comfort of the families of the patient (the hospitalization process is shown in Figure 2).

\section{DISCUSSION}

In the previous studies, the fungal infections of the CNS were rare clinical cases, but candidemia cases have increased dramatically worldwide $(8,9)$. Fungal infections can 
develop via hematogenous dissemination from a distant focus such as central venous catheter and lung (10). The symptoms of Candida meningitis are often similar to those in patients with bacterial meningitis such as fever (hyperthermia), headache, neurological symptoms, and meningeal irritation (11).

According to the results of the current research (7), a high index of suspicion is needed in any patient with risk factors such as abdominal surgery, bowel perforation, recent broadspectrum antibiotic therapy, intravenous drug use, advanced in years, indwelling catheters, low immune function, severe burns, antineoplastic therapy, and long-term glucocorticoid usage $(12,13)$. The development of fungal infections largely depend on the interplay between the health status of the patients and the virulence of pathogenic fungal species. In many cases, there is no specificity in the clinical symptoms. As we know, fungal meningitis is a common complication of immunosuppression, while Candida albicans is the most common pathogen $(14,15)$. Overall, albicans were the most common strains followed by Candida glabrata in the clinical cases, accounting for about half of the total cases, but the source of candidemia was unknown in half of all the episodes (16).

In this case, the patient lacked any known risk factors, and the reason for the infection needed further study. According to the current diagnosis, fungal infection can be isolated from blood and CSF, which is the gold standard of the clinical diagnosis. However, the positive rate in the blood cultures and CSF is too low for the clinician. Even if the etiological culture is positive, it will take 3-5 days at the fastest. With respect to the timing of initiation of empirical treatment, some studies are still controversial, with some showing that mortality was lower in the patients who received the antifungal early $(7,17)$, while other studies showed that there was no significant difference in in-hospital mortality $(18,19)$.

With most of the research results, the prompt initiation of the antifungal therapy was essential for better outcomes. But in any case, timely and effective treatment with the appropriate antiinfective drugs is the most crucial factor for the best prognosis (20). Providing effective treatment for the patients is the most crying need for the patients and doctors. Meanwhile, if we can get accurate results earlier, the therapeutic efficacy will be better and fewer irrational antifungal drugs will be used. However, in most of the cases, the clinicians did not have adequate laboratory tests at the beginning; they can only judge the condition according to the guidelines, expert consensus, and clinical experience, so as to minimize the possibility of missed diagnosis, misdiagnosis, and overdiagnosis and treatment $(3,7)$.

The incorrect usage of the antimicrobials will cause the resistance of the pathogen, treatment difficulty, and the tensional doctor-patient relationship, especially in China (21). Fungal cultures of CSF have a bad sensitivity and this method is rarely helpful. Due to the poor sensitivity of the cultures, the diagnosis of fungal infection is very tricky (22). In addition, unlike the other infection, there is not a widely accepted molecular-, antigen-, or nucleic acid-based testing method to expedite the identification of the organism in the medical units, thus delaying the proper treatment (23). Now, diagnoses of these diseases are still tricky because there lacked a fast and reliable means of detection. Delay in the diagnosis and inappropriate therapy can result in permanent damage, even death. It was an unacceptable outcome.

Clinical doctors need another simple and effective method to isolate and detect the pathogen. In the current practice, culture-based methods are still considered as the standard approach of etiological diagnosis of the infection. However, these methods have disadvantages including long turnaround time and practically low sensitivity. Although molecular assays such as PCR-based methods have been established for the pathogen, the detection is limited to the known pathogens listed on the panel.

The NGS is a new laboratory detection technology that directly extracts the nucleic acids from all the pathogens in the samples of the patient for high-throughput sequencing; the NGS is able to detect the thousands of fragments in a laboratory test, therefore this technology can reduce the number of tests and period of detection (24). In the past few years in China, NGS is an emerging innovative technology, which has become highly sensitive in the detection of pathogens (24). NGS has been constantly improving and stepped into clinical practice, providing a powerful tool with advantages including short turnaround time, full-spectrum, and semiquantitative analysis. In comparison to the routine cultural method, NGS has made a huge advancement in infection diagnosis. NGS had become a new and innovative technology and is gradually applied in clinical practice. In this case, the NGS results showed that pathogens were Candida parapsilosis and Candida orthopsilosis. They are the different genotypes of Candida (25), but only Candida parapsilosis is stated in this clinical guideline (3). This also proved the advantages of NGS (19).

The antimicrobial resistance profile of Candida in China showed that the isolates were sensitive to the liposomal amphotericin B and fluconazole. This is consistent with the IDSA guidelines (5) and this case indirectly proved the sensitivity and specificity of NGS.

The limitations included few acknowledged criteria of the sequencing result explanation which have been established (26); large-scale study comparing NGS with traditional etiological diagnosis methods which are missing, the sample size of most of the studies is small (27); this study is just a case report, thus, it still needs high-level evidence to support its clinical application; and this method is very sensitive, so strict aseptic operation is required in the detection process, else it is easy to lead to the clinical misdiagnosis (28).

\section{CONCLUSION}

Next-generation sequencing technology is a new method of diagnosis. Based on the unique advantages of the NGS technology in the diagnosis of these infectious diseases, this new technology will gradually be applied in clinical practice to benefit a large number of patients. 


\section{DATA AVAILABILITY STATEMENT}

The datasets presented in this study can be found in online repositories. The names of the repository/repositories and accession number(s) can be found at: Genome Sequence Archive [BioProject: PRJCA006605/CRP003428; https://bigd.big.ac.cn/ gsa/browse/CRA005014].

\section{REFERENCES}

1. Donnelly JP, Chen SC, Kauffman CA, Steinbach WJ, Baddley JW, Verweij PE, et al. Revision and update of the consensus definitions of invasive fungal disease from the European Organization for Research and Treatment of Cancer and the Mycoses Study Group Education and Research Consortium. Clin Infect Dis. (2019) 6:1367-76. doi: 10.1093/cid/ci z1008

2. Bassetti M, Garnacho-Montero J, Calandra T, Elie A, Arunaloke C, Daniel $\mathrm{K}$, et al. Intensive care medicine research agenda on invasive fungal infection in critically ill patients. Intensive Care Med. (2017) 43:122538. doi: 10.1007/s00134-017-4731-2

3. Pappas PG, Kauffman CA, Andes DR. Clinical practice guideline for the management of candidiasis: 2016 update by the infectious Diseases Society of America. J Clin Infect Dis. (2016) 62:e1-50. doi: 10.1093/cid/c iv933

4. Goemaere B, Becker P, Wijngaerden EV, Maertens J, Spriet I, Hendrickx M, et al. Increasing candidemia incidence from 2004 to 2015 with a shift in epidemiology in patients pre-exposed to antifungals. Mycoses. (2017) 61:12733. doi: $10.1111 /$ myc. 12714

5. Góralska K, Blaszkowska J, Dzikowiec M. Neuroinfections caused by fungi. Infection. (2018) 46:443-59. doi: 10.1007/s15010-018-1152-2

6. Bridges $\mathrm{KJ}$, Li R, Fleseriu M, Cetas JS. Candida meningitis after transsphenoidal surgery: a single-institution case series and literature review. World Neurosurg. (2017) 108:41-9. doi: 10.1016/j.wneu.2017. 08.115

7. Stoeter DJ, Michael BD, Solomon T, Poole L. Managing acute central nervous system infections in the UK adult intensive care unit in the wake of UK encephalitis guidelines. J Intensive Care Soc. (2015) 16:3308. doi: $10.1177 / 1751143715587927$

8. Camargo T, Marra AR, Silva CV, Cardoso MF, Martino MD, Camargo LF, et al. Secular trends of candidemia in a tertiary care hospital. Am J Infect Control. (2010) 38:546-51. doi: 10.1016/j.ajic.2009. 12.012

9. Wisplinghoff H, Bischoff T, Tallent SM, Seifert H, Wenzel RP, Edmond MB. Nosocomial bloodstream infections in US hospitals: analysis of 24,179 cases from a prospective nationwide surveillance study. Clin Infect Dis. (2004) 39:309-17. doi: 10.1086/421946

10. Mohan S, Jain KK, Arabi M, Shah GV. Imaging of meningitis and ventriculitis. Neuroimaging Clin N Am. (2012) 22:557-83. doi: 10.1016/j.nic.2012.04.003

11. de Gans J, van de Beek D. Dexamethasone in adults with bacterial meningitis. Neuroimaging Clin N Am. (2012) 22:557-83. doi: 10.1016/j.nic.2012.4.003.

12. Guillermo Q. Epidemiology of candidaemia and invasive candidiasis. A changing face. Rev Iberoam Micol. (2014) 31:428. doi: 10.1016/j.riam.2013.10.001

13. Barter DM, Johnston HL, Williams SR, Tsay SV, Vallabhaneni S, Bamberg WM. Candida bloodstream infections among persons who inject drugs - Denver metropolitan area, Colorado, 2017-2018. MMWR Morb Mortal Wkly Rep. (2019) 68:285-88. doi: 10.15585/mmwr.mm $6812 \mathrm{a} 3$

14. Miranda EJ, Gonçalves LG, Oscar DS. Cryptococcal meningitis in HIV-negative patient with liver cirrhosis due to hepatitis C. Braz $J$ Infect Dis. (2011) 15:399-00. doi: 10.1590/S1413-867020110004 00019

15. Tan BH, Chakrabarti A, Li RY, Watcharananan SP, Liu Z, Chindamporn $\mathrm{A}$, et al. Incidence and species distribution of candidaemia in Asia: a

\section{AUTHOR CONTRIBUTIONS}

CY conceived the idea of this article. XC and CW have made the same contributions to the article and they contributed to the preparation of the manuscript and interpreting the data of the patients. All the authors contributed to review, edit, read, and approved the final manuscript.

laboratorybased surveillance study. Clin Microbiol Infect. (2015) 21:94653. doi: 10.1016/j.cmi.2015.06.010

16. Yeșilkaya A, Azap Ö, Aydin M, Mehtap AO. Epidemiology, species distribution, clinical characteristics and mortality of candidaemia in a tertiary care university hospital in Turkey, 2007-2014. Mycoses. (2017) 60:4339. doi: $10.1111 /$ myc. 12618

17. Abbas $\mathrm{H}$, Cifuentes KA, Abbas N, Cindrich R, Singh M. A rare presentation of Cryptococcal meningitis and cerebellitis in an asplenic patient, seronegative for Human Immunodeficiency Virus (HIV). Am J Case Rep. (2018) 19:18386. doi: 10.12659/AJCR.906869

18. Bassetti M, Righi E, Ansaldi F, Rocca DG, Antonelli M, Tumbarello $\mathrm{M}$, et al. A multicenter study of septic shock due to candidemia: outcomes and predictors of mortality. Intensive Care Med. (2014) 40:83945. doi: 10.1007/s00134-014-3310-z

19. Kludze-Forson M, Eschenauer GA, Kubin CJ, Della-Latta P, Simon WL. The impact of delaying the initiation of appropriate antifungal treatment for Candida bloodstream infection. Med Mycol. (2010) 48:436-39. doi: 10.3109/136937809032 08256

20. Ryuichi H, Yuichi S, Junichi K, Yamamoto S, Kayaba H. Epidemiology, practice patterns, and prognostic factors for candidemia; and characteristics of fourteen patients with breakthrough Candida bloodstream infections: a single tertiary hospital experience in Japan. Infect Drug Resist. (2018) 31:821-33. doi: 10.2147/IDR.S1 56633

21. Wei P, Gan D, Qi T, Xu LZ. Continuing violence against medical personnel in China: a flagrant violation of Chinese law. Biosci Trends. (2016) 19:24043. doi: 10.5582/bst.2016.01094

22. Black KE, Baden LR. Fungal infections of the CNS: treatment strategies for the immunocompromised patient. CNS Drugs. (2007) 21:293-18. doi: 10.2165/00023210-20072104000004

23. Li L, Wang L, Zhang C, Chen P, Luo X. A case of Vibrio vulnificus related wound infection diagnosed by next-generation sequencing. ID Cases. (2019) 24:e00497. doi: 10.1016/j.idcr.2019.e00497

24. Alekseyev YO, Fazeli R, Yang S, Basran R, Miller NS, Remick D, et al. A next-generation sequencing primer-how does it work and what can it do? Acad Pathol. (2018) 5:2374289518766521. doi: 10.1177/23742895187 66521

25. Arastehfar A, Khodavaisy S, Daneshnia F, Weihua P, Ferry H, Teun B, et al. Molecular identification, genotypic diversity, antifungal susceptibility, and clinical outcomes of infections caused by clinically underrated yeasts, Candida orthopsilosis, and Candida metapsilosis: an Iranian multicenter study (20142019). Front Cell Infect Microbiol. (2019) 9:264. doi: 10.3389/fcimb.2019. 00264

26. Zhong $\mathrm{Y}, \mathrm{Xu} \mathrm{F}, \mathrm{Wu}$ J, Marilyn ML. Application of next generation sequencing in laboratory medicine. Ann Lab Med. (2021) 41:25-43. doi: 10.3343/alm.2021.41.1.25

27. Wilson MR, Sample HA, Zorn KC, Gabriela M, Randall H, Kevin M, et al. Clinical metagenomic sequencing for diagnosis of meningitis and encephalitis. N Engl J Med. (2019) 380:2327-40. doi: 10.2147/IDR.S56633

28. Thoendel M, Jeraldo P, Greenwood-Quaintance KE, Yao J, Chia N, Hanssen $\mathrm{AD}$, et al. Impact of contaminating DNA in whole-genome amplification kits used for metagenomic shotgun sequencing for infection diagnosis. J Clin Microbiol. (2017) 55:1789-801. doi: 10.1128/JCM.02 402-16 
Author Disclaimer: All the claims expressed in this article are solely those of the authors and they do not necessarily represent those of their affiliated organizations or those of the publisher, the editors, and the reviewers. Any product that may be evaluated in this article, or claim that may be made by its manufacturer, is not guaranteed or endorsed by the publisher.

Conflict of Interest: The authors declare that the research was conducted in the absence of any commercial or financial relationships that could be construed as a potential conflict of interest.

Publisher's Note: All claims expressed in this article are solely those of the authors and do not necessarily represent those of their affiliated organizations, or those of the publisher, the editors and the reviewers. Any product that may be evaluated in this article, or claim that may be made by its manufacturer, is not guaranteed or endorsed by the publisher.

Copyright $\odot 2021 \mathrm{Cao}, \mathrm{Yu}$, Zhou, Huang and Wang. This is an open-access article distributed under the terms of the Creative Commons Attribution License (CC BY).

The use, distribution or reproduction in other forums is permitted, provided the original author(s) and the copyright owner(s) are credited and that the original publication in this journal is cited, in accordance with accepted academic practice. No use, distribution or reproduction is permitted which does not comply with these terms. 